Dhaka Univ. J. Biol. Sci. 19(2): 171-179, 2010 (July)

\title{
DEVELOPMENTAL STAGES OF A MOTTLED EMIGRANT BUTTERFLY, CATOPSILIA PYRANTHE
}

\author{
M. H. ArJu, M. A. Bashar And G. Moula \\ Department of Zoology, University of Dhaka, Dhaka-1000, Bangladesh
}

Key words: Life cycle, Catopsilia pyranthe, Developmental stages, Rearing

\begin{abstract}
The overall life cycle, duration and survival rate of different developmental stages (egg, larva and pupa) of Catopsilia pyranthe conducted at $27 \pm 3^{\circ} \mathrm{C}$ and $\mathrm{RH}$ $75 \% \pm 2$. showed that within 6.4 days $68 \%$ eggs hatched. The average duration from egg to adult, larval stage and pupal were 23.87, 10.93 and 6.8 days, respectively. Fifty six out of 68 larvae successfully completed their whole 5 instars. There were positive correlation among the larval instars, amount of food consumption and excretion of faeces. About $52 \%$ pupa were emerged as adult at laboratory condition.
\end{abstract}

\section{Introduction}

A successful and effective conservation management of butterflies depend on sound knowledge of their life history and host plant requirements in the wild state. Butterflies are particularly sensitive to environmental variations.(1) Positive relations have been found between butterfly diversity and environmental variables, such as plant diversity(2-5) habitat complexity, ${ }^{(6)}$ landscape structure, ${ }^{(7)}$ topographic and moisture gradients ${ }^{(8)}$ and climate. ${ }^{(9,10)}$

Butterflies are very much related with their host-plants. It is believed that the conservation of butterflies is related to the conservation of host-plant species. It is estimated that there are more than 17,500 butterfly species in the world and 90 per cent of them have been described. (11) Pieridae is the third largest butterfly family of the world with 2000 described species. ${ }^{(12)}$ They are typically active during the day. Seven families of butterflies, such as Papilionidae, Pieridae, Nymphalidae, Danaidae, Satyridae, Lycaenidae and Hesperiidae are found in Bangladesh. Among the seven families only Pieridae and Nymphalidae have been taxified and identified up to species level on the basis of wing-venation in Bangladesh. ${ }^{(13-15)}$

The genus Catopsilia is a member of the family Pieridae and sub-family Coliadinae (Lepidoptera: Rhopalocera). It is a common butterfly found all over in Bangladesh. Although Beeson (16) mentioned four species of the genus Catopsilia, namely C. pomona, C. pyranthe, C. florella and C. crocale, but Varshney ${ }^{(17)}$ recognized only two species as $C$. pyranthe (Linn.) with florella (Fab.) as the dry-season form and C. crocale (Cramer) with 
two separate subspecies C. Pomona and C. crocale. They are common throughout the year but the monsoon promoting higher frequency of population. ${ }^{(18)}$

Earlier, it was reported that the Catopsilia pyranthe breeds throughout the year and the developmental period from egg to adult requires 22 to 29 days allowing up to $11-12$ generations a year.(19) Host plants included Cassia spp. (e.g. C. fistula, C. javanica, C. roxbarghii, C. bakeriana), Ganidia glauca, Senna spp.(20)

\section{Materials and Methods}

It is well-known that the Catopsilia pyranthe lay eggs on Cassia fistula, C. alata, C. javanica, C. auriculata and Albizia lebbeck. During the present experiment. eggs were collected from Cassia javanica leaves at the Curzon Hall campus of the University of Dhaka. The eggs were collectively connected with the branch of host plant, which were collected by cutting the stem at $45^{\circ}$ angle. Immediately after cutting, soaked cotton was set up at the end of cutting stem. Water was sprayed as and when necessary then placed it onto the rearing cages maintaining $24-30^{\circ} \mathrm{C}$ temperature of about appropriate relative humidity. Ten eggs were placed on each cage. Two sets of rearing cages were used in this experiment. Each rearing cage had three edges. The length of first (Fig. 1c), second (Fig. 1d) and third (Fig. 1e) edge of rearing cage were 14.5, 14 and 14 inches, wide were 12, 11.5 and 11.5 inches and the height were 6,5 and 2.5 inches, respectively. First edge had
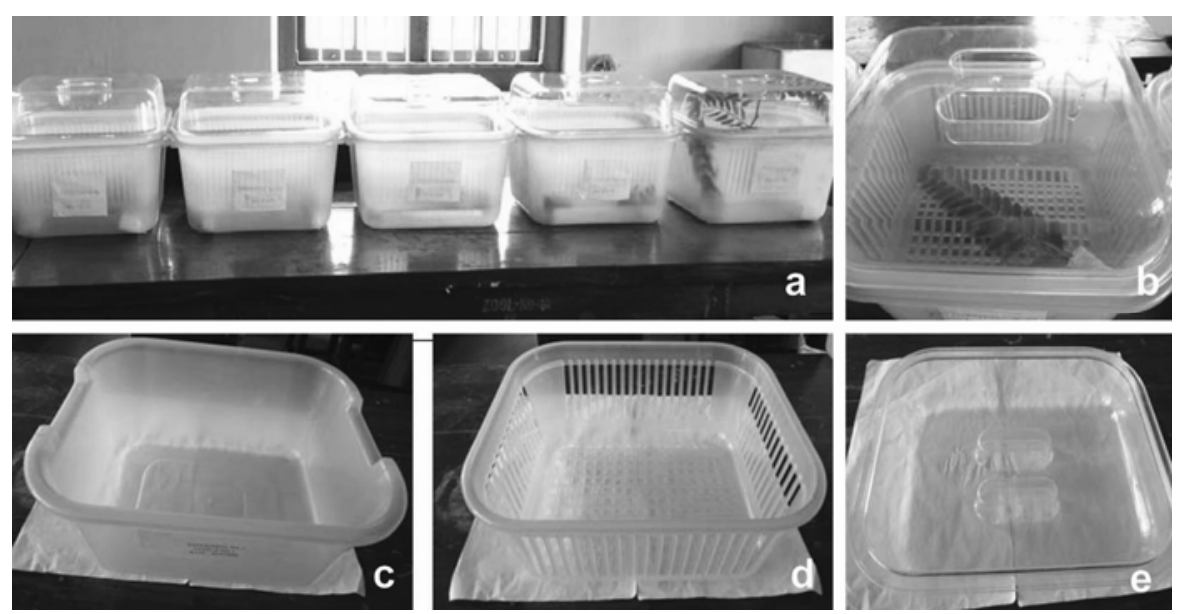

Fig. 1. Rearing cages. (a) Arrangement of experimental cages. (b) Host plant leaves and larva in an experimental cage. (c) First edge of the rearing cage. (d) Second edge of the rearing cage, (e) Third edge of the rearing cage.

no cavity, second edge was fully perforated and the third edge was the cover of the cage. These cages were used for the maintenance of proper humidity and limited air supply. The experimental cages were placed in the Environmental Biology and Biodiversity 
Laboratory (EBBL), Department of Zoology, University of Dhaka (Fig. 1a). The room temperature was $27 \pm 3^{\circ} \mathrm{C}$ and $\mathrm{RH}$ was $75 \pm 2 \%$. Eggs were examined twice per day. The eggs with its host plant were covered to reduce desiccation.

Larvae were kept in the same rearing cages. Larvae were observed regularly for supplying food, collecting faeces and to identify the molting and mortality rate. Larval length was measured by using centimeter scale. After completion of measurement larvae were transferred into another set of rearing cage. Fresh leaves of Cassia javanica were supplied as food at each two days interval for first instars and one day interval for second instars. Old foliage was removed the following days after larvae migrated to a new leaf. After that it was placed onto the second perforated edge of the rearing cage. The host plant was kept fresh by watering. After that the second perforated edge was placed onto the first edge of the rearing cage and then covered by the third edge (Fig. 1b). Injured or damaged leaves were avoided for rearing of larvae. Comparatively third, fourth and fifth instar larvae were feed voraciously and produced a large amount of faeces. Fresh leaves of Cassia javanica were supplied daily for them. Leaves were replaced when more than half of the leaf was eaten. Brush was used to clean the faeces from the cages regularly. The amount of food consumption and faeces (gm) were recorded by using precision electronic balances. Larval duration was also observed very carefully. Their feeding potential was measured following the method of Barua and Slowik and Singh and Singh. ${ }^{(21,22)}$ All the equipment like brush, pair of forceps, sprayer, scissor etc. were sterilized by following the soapy water and autoclaving.

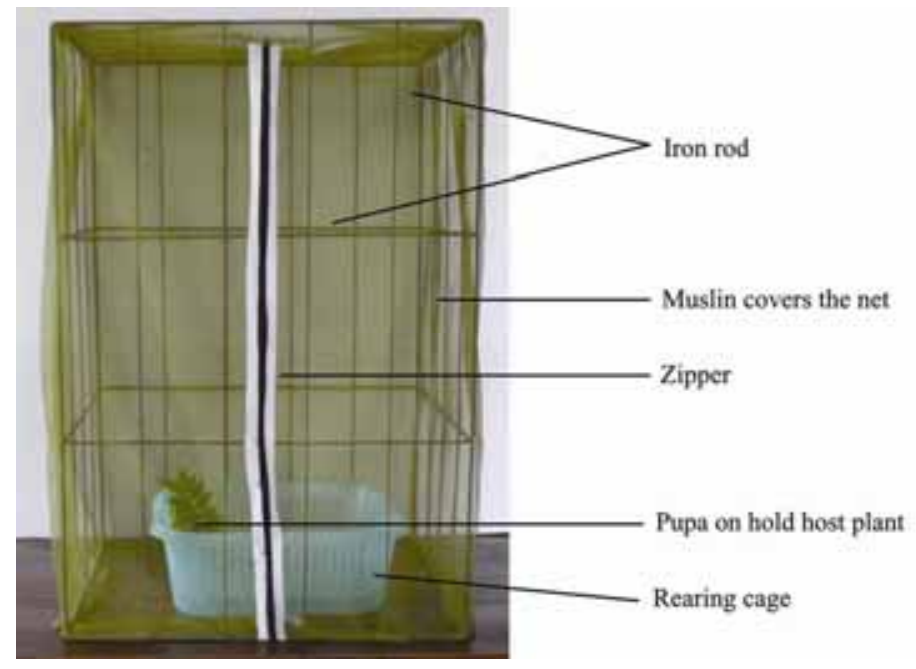

Fig. 2. Emergence cabinet.

Pupa with rearing cages were kept in the emergence cabinet (Fig. 2). This cabinet was made by iron rod. It was rectangular in shape (Length 21 inch, width 18 inch, and height 36 inch). It was covered by the muslin net to prevent the butterfly dispersion or flight 
when the adult emerged from the pupa. There were $8-10$ holes per $1 \mathrm{~cm}^{2}$ area in net for maintaining the proper light and aeration. A zipper was attached to the muslin net before it used to cover.

Damp sponges were kept in the immergence cabinet at the period of adult emerge for providing sufficient humidity. The adults were preserved in the laboratory for further experiment.

\section{Results and Discussion}

Different stages of the lifecycle of Catopsilia pyranthe are presented in Fig. 3.

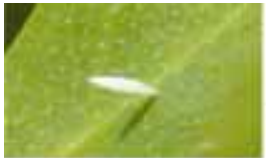

(a) Egg

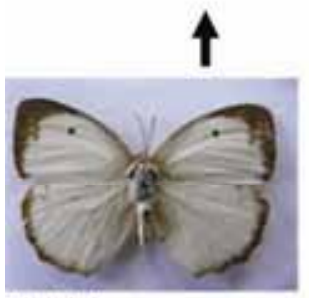

(j) Adult

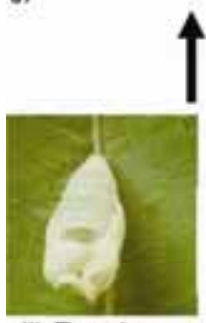

(i) Exuviae
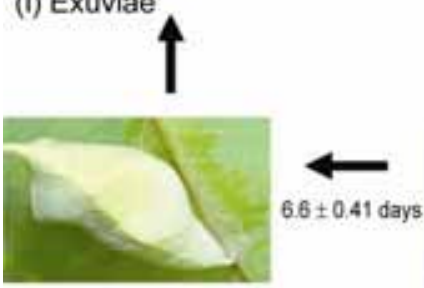

(h) Pre emergence stage

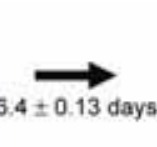

(b) 1st instar larva

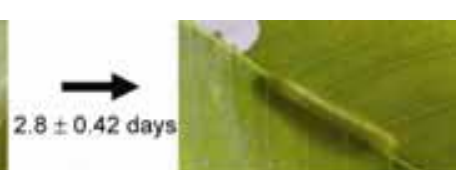

(c) 2nd instar larva

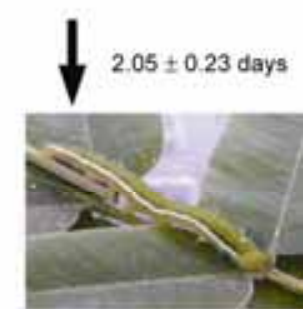

(d) 3rd instar larva $2.11 \pm 0.31$ days

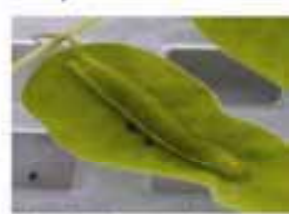

(e) 4th instar larva $\downarrow$ $2.05 \pm 0.23$ days

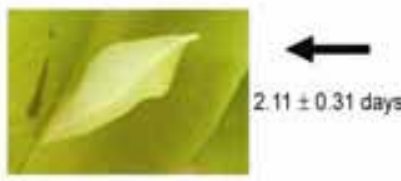

(g) Pupa

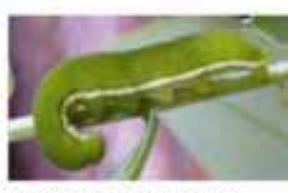

(f) 5 th instar larva

Fig. 3. Different stages of life cycle of Catopsilia pyranthe

Eggs (Fig. 3a) were erect and spindle in shape and measuring about $2 \mathrm{~mm}$ in height and $1 \mathrm{~mm}$ in diameter at the broadest region. Eggs consist of a hard ridged outer layer of 
shell, called the chorion. A thin coating of wax present in chorion which prevents eggs from drying out.

Incubation period of $C$. pyranthe was studied in laboratory at $27 \pm 3^{\circ} \mathrm{C}$ temperature with RH 75 \pm 2 . During the study observation was made on the changement of egg colouration, embryo inside the egg and finally nature of larval hatching. The duration of incubation period (in days) were recorded very carefully. The colour of egg was found white immediately after hatching, which becoming creamy white within a day. It became dark white at the time of hatching. The full formed embryo was clearly visible within the transparent egg shell during just before hatching. By splitting the transparent egg shell longitudinally, the young larva gnaws and came out.

No larvae were recorded to hatch before passing six days as incubation period. Some of them were found to hatch after six and half days passed in the period. No one was recorded to hatch after seven days as incubation period. About $49 \%$ of the egg-hatch was found to occur on 6 th day $30 \%$ on 6.5 th day and $21 \%$ on 7 th day. So, maximum duration was 7.0 days with minimum 6.0. The average period was 6.4 days (Fig. 4).

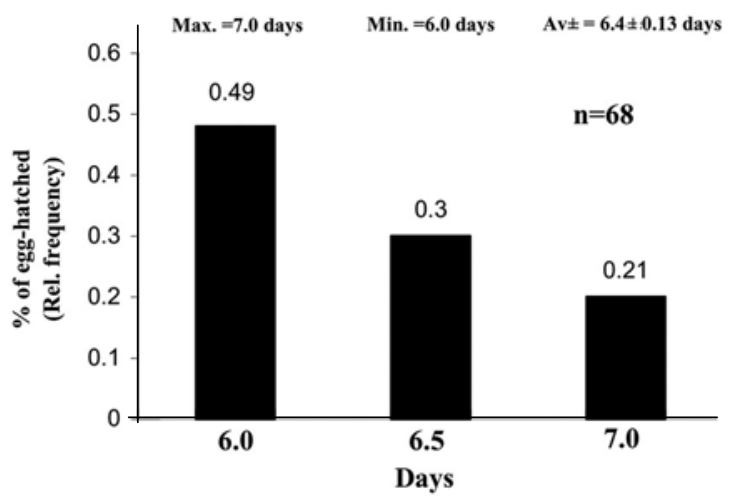

Fig 4. Rate of incubation period (in days) of C. pyranthe in Laboratory at RH $75 \% \pm 2$ and $\left.27 \pm 3^{\circ} \mathrm{C}\right)$.

About 56\% larvae completed its whole five instars. Freshly hatched larva feed its own egg shell first. It grow throw a series of moults and each intermediate stage is called an instar. The larva of $C$. pyranthe moult usually four times. During moulting the larvae stop feeding and take rest. First, second, third, fourth and fifth larval instar duration were two - three days and body length were 3 - 8, 9 - 17, 18 - 28, 29 - 34 and 35 - $45 \mathrm{~mm}$, respectively.

First instar larva (Fig. 3b): First instar larva was very tiny. Body shape was cylindrical and pale green in colour. Body distinct into head, thorax and anal region. Yellow abdominal prolegs were also observed. Head was rounded and plain and it was 0.3 to 0.5 $\mathrm{mm}$ in diameter. This instar fed very slowly. 
Second instar larva (Fig. 3c): Colour pale green and lateral sides yellow. Head was pale yellow, 0.6 to $1.3 \mathrm{~mm}$ in diameter. Body segments were slightly visible. Larva fed slowly and excreted a very less amount of faeces which was black in colour and alike to dust.

Third instar larva (Fig. 3d): Head 1.4 to $1.7 \mathrm{~mm}$ in diameter. Body green in colour, a distinct black band was observed just above the white lateral line. Legs and prolegs were very distinct. In this stage larva fed very fast and grew rapidly. It excreted a huge amount of faeces which was black in colour and granular.

Fourth instar larva (Plate 3,e): Green in colour. Head rounded, yellowish green and 1.8 to $2.5 \mathrm{~mm}$ in diameter. In this instar larva consumed a huge amount of host plants leaves. Remaining characters were identical to third instar.

Fifth instar larva (Fig. 3f): At this stage larva consumed the leaves as their food voraciously. Body was as like as fourth instar. Three segments were observed in thorax and each segment a pair of true legs was observed. Ten segments were found in abdomen and each segment from three to six a pair of false legs or prolegs were present. Anal prolegs were also observed in the last segment. In this instar larva excreted a huge amount of faeces.

Fig. 5 shows the total duration of larval period. No larvae turned into pupa before ten days. Some of them turned into pupa after 11 days and no one was recorded to turn into pupa after 12 days. Among the larvae $23.21 \%$ were found to turn into pupa on 10 th day, $60.71 \%$ on 11 th day and $16.07 \%$ on 12 th day. Maximum duration was recorded 12 days and minimum was ten days.

There was a positive correlation between the number of larval instar and the amount of food consumption. The maximum food consumed was $2.12 \mathrm{~g}$ in fifth instar and minimum was $0.10 \mathrm{~g}$ in first instar (Fig. 6)

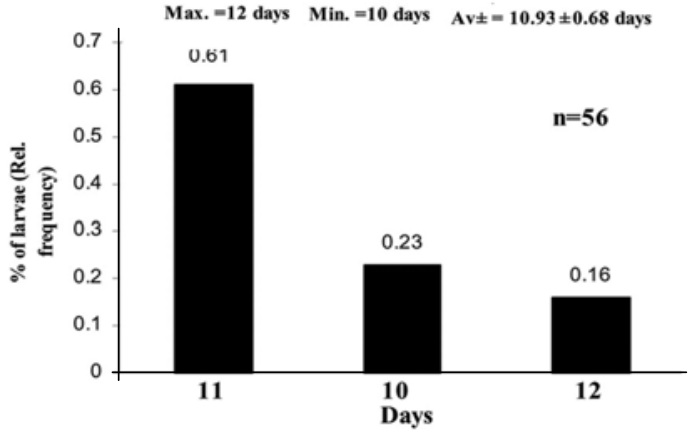

Fig. 5. Total duration of larval period of $C$. pyranthe in laboratory at $27 \pm 3^{\circ} \mathrm{C}$ and $\mathrm{RH} 75$ $\pm 2 \%$ ).

There was also a positive correlation among the larval instar and the amount of excreted faeces. The highest amount of faeces was recorded at $0.44 \mathrm{gm}$ in fifth instar and lowest was $0.01 \mathrm{gm}$ in first instar (Fig. 7). 
Pre-pupal stage: Body becomes thick and short by contraction and was recorded 30 to $35 \mathrm{~mm}$ in length. Then they attach themselves to the lower side of the leaf.

Pupal stage (Fig. 3g): It was pale green in colour with a yellow lateral line. It was totally inactive. It had a hard protective coating. It shown camouflage to evade potential predators.

No pupa was emerge before passing six days and after seven days as pupation. Among them $32.70 \%$ pupae were found to emerge on 6th day, $11.54 \%$ on 6.5 th day and $55.77 \%$ on seventh day. The average period of emerge was recorded 6.62 days with the Sd of \pm 0.41 . The maximum duration was recorded 7.0 days and minimum was 6.0 days (Fig. 8).

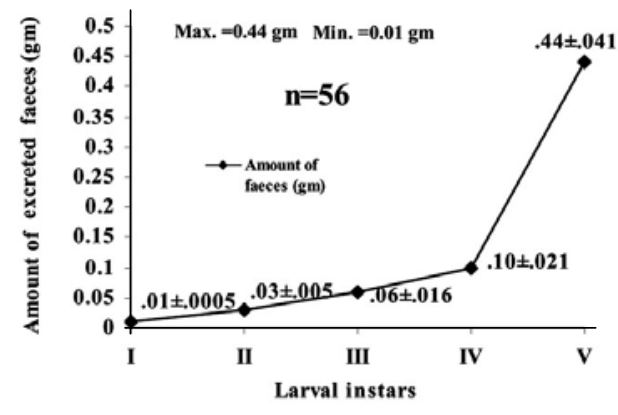

Fig. 7. Amount of excreted faeces (in g) of each larval instar of $C$. pyranthe.

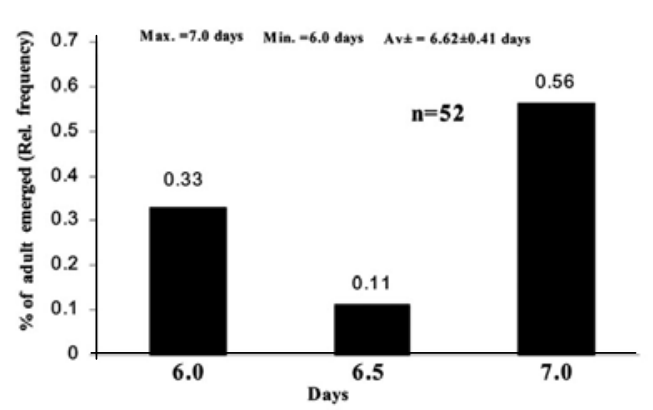

Fig. 8. Rate of pupal period (in days) of $C$. pyranthe in laboratory ( $\mathrm{RH} 75 \pm 2 \%$ and $27 \pm$ $\left.3^{\circ} \mathrm{C}\right)$.

Pre-emergence stage (Fig. 3h): Wings and head were visible from the outside of the pupal case during the period of emergence. This time they excreted yellow fluid.

Emergence of butterfly: Catopsilia pyranthe emerge (eclose) from the pupa by splitting the pupal case (Fig. 3i). After emerges from pupa it did not fly until the wings were unfolded. Newly emerged butterfly needed to spend some time inflating its wings with blood and letting them dry. Its wings took up to two to three hours to dry.

During the study of $C$. pyranthe in laboratory the total duration from egg to adult emerge was recorded 22 to 26 days. No adult was emerged before 22 days and after 26 days. About $30 \%$ were found to emerge on 24 th day, $12 \%$ on 23 rd day, $9 \%$ on 24.5 th day, $9 \%$ on 23.5 th day, $8 \%$ on 26 th day, $8 \%$ on 25.5 th day, $8 \%$ on 25 th day, $8 \%$ on 22.5 th day and $8 \%$ on 22 nd day. So, maximum duration was 26 days with minimum 22 days. The average duration was 23.87 days with the $\mathrm{Sd}$ of \pm 0.98 days (Fig. 9).

The total survival rate from egg to adult was recorded 52\%. Among 100 individuals survival rate of egg was recorded $68 \%$. Overall $32 \%$ egg did not hatch. Nineteen of those eggs became moldy at five to six days after collection and other 13 eggs developed mold 
in 8 - 11 days. Survival rate of larva was recorded 56\%. Twelve died at first instar (4 was deformed so that it could not feed properly and 8 became diseased). Survival rate of pupa was $52 \%$. Four pupae was not emerged, they became mold after 7 days. So, survival rate was highest in pupal stage and it was lowest in egg-hatch (Fig. 10).

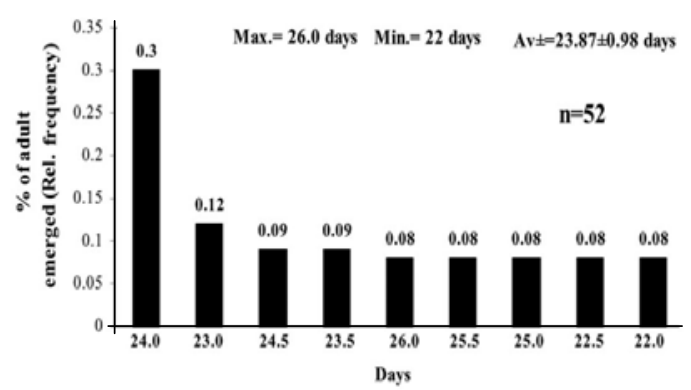

Fig. 9. Total duration from egg to adult of $C$. pyranthe in laboratory (at $27 \pm 3^{\circ} \mathrm{C}$ and $\mathrm{RH}$ $75 \pm 2 \%)$

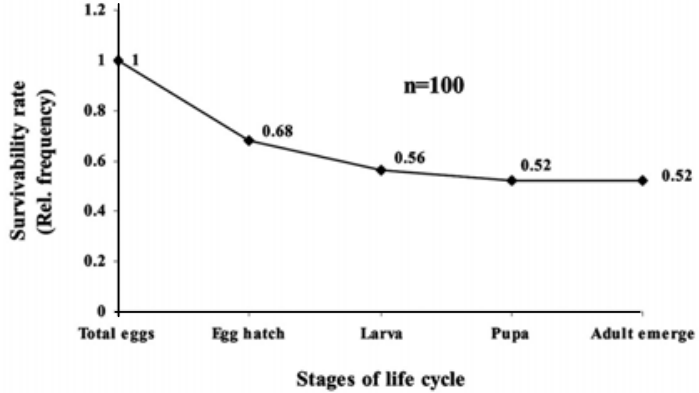

Fig. 10. Survivability of C. pyranthe in different developmental stages (Egg, larva, pupa and adult).

Adult (Fig. 3j): Adult wing span was 46 to $60 \mathrm{~mm}$ (Fore wing span $25-30 \mathrm{~mm}$ and hind wing span $20-23 \mathrm{~mm}$ ). Antennae were red and 10-12 mm in length. Upper side was greenish white. Female has broader black apical margins and traces of a black costal border on the fore wing. Hind wing also have a black border. The male has black apical border on the forewing, and no marking on the hind wing.

\section{References}

1. Scoble MJ 1992. The Lepidoptera: Form, Function and Diversity. Oxford University Press, New York. pp. 253.

2. Erhardt A. 1985. Diurnal Lepidoptera: Sensitive indicators of cultivated and abandoned grassland. J. Appl. Ecol. 22: 849-861.

3. Thomas CD and HC Malorie 1985. Rarity, species richness and conservation: Butterflies of the atlas mountains in Morocco. Biological Conservation 33: 95-117.

4. Leps J and K Spitzer 1990. Ecological determinants of butterfly communities (Lepidoptera, Papilionidae) in the Tam Dao Mountains, Vietnam. Acta Entomologica Bohemoslovaca 87: 182-194.

5. Spitzer K, J Jaros, J Havelka and J Leps 1997. Effect of small-scale disturbance on butterfly communities of an Indochinese mountain rainforest. Biological Conservation 80: 9-15.

6. Molina JM and JM Palma 1996. Butterfly diversity and rarity within selected habitats of western Andalusia, Spain (Lepidoptera: Papilionoidea and Hesperiodea). Nota Lepidopterologica 78: 267-280.

7. Wood PA and MJ Samways 1992. Landscape element pattern and continuity of butterfly flight paths in an ecologically landscaped botanic garden, Natal, South Africa. Biological Conservation 58: 149-166. 
8. Kremen C 1992. Assessing the indicator properties of species assemblages for natural areas monitoring. Ecological Applications 2: 203-217.

9. Pollard E and TJ Yates 1993. Monitoring Butterflies for Ecology and Conservation. Chapman \& Hall. London. pp. 443.

10. Parmesan C 1996. Climate and species range. Nature 382: 765-766.

11. Robbins RK and PA Opler 1997. Butterfly diversity and a preliminary comparison with bird and mammal diversity. Joseph Henry Press, Washington D. C. pp. 69-82.

12. Fres PS 1989. The Illustrated Encyclopedia of the Butterfly World. Salamander Books Ltd. pp. 1-275.

13. Bashar MA, MA Mamun and SKM Rahaman 2005. Wing-venation as a factor for the identification of Pierid butterflies in the forests of Bangladesh. Bangladesh J. Zool. 33(1): 49-56.

14. Bashar MA, MA Mamun, AFM Aslam and AK Chowdhury 2006a. Biodiversity maintenance and conservation of butterfly-plant association in some forests of Bangladesh. Bangladesh J. Zool. 34(1): 55-67.

15. Bashar MA, MA Mamun, HR Khan and AK Chowdhury 2006b.Wing-venation as a factor for the identification of Nymphalid butterflies in the forests of Chittagong and Cox's Bazar (Bangladesh). Bangladesh J. Zool. 34(2):187-193.

16. Beeson LFC 1941. The ecology and control of the forest insects of India and the neighboring countries. Govt. of India.

17. Varshney RK 1980. J. Bombay Nat. Hist. Soc. 76: 33-40.

18. Wynter BMA 1957. Butterflies of the Indian Region. J. Bombay Nat. Hist. Soc.

19. Atluri, JB, SPV Ramana and CS Reddi 2004. Ecobiology of the tropical pierid butterfly Catopsilia pyranthe. Current Science. 86(3): 2.

20. Kunte K 2006. Additions to known larval host plants of Indian butterflies. J. Bombay Nat. His. Soc. 103(1): 119-120.

21. Barua KK and J Slowik 2007. Study on the biology and consumption potential of common rose, Pachliopta aristolochiae aristolochiae (Lepidioptera: Papilionidae) on Aristolochia tagala. Polish J. Ent. 76: 341-352.

22. Singh I and G Singh 1993. Assessment of foliage loss caused by different larval instars of bihar hairy caterpillar, Spilosoma obliqua (Walker) on sunflower. J. Insect. Sci. 6(2): 185-186. 